Köller

Philosophie der Grammatik 
Wilhelm Köller

\title{
Philosophie der Grammatik
}

\author{
Vom Sinn \\ grammatischen Wissens
}

J.B. Metzlersche

Verlagsbuchhandlung

Stuttgart 
CIP-Titelaufnahme der Deutschen Bibliothek

Köller, Wilhelm:

Philosophie der Grammatik : vom Sinn grammat. Wissens / Wilhelm Köller. - Stuttgart : Metzler, 1988

ISBN 978-3-476-00632-5

ISBN 978-3-476-00632-5

ISBN 978-3-476-03252-2 (eBook)

DOI 10.1007/978-3-476-03252-2

Dieses Werk einschließlich aller seiner Teile ist urheberrechtlich geschützt.

Jede Verwertung außerhalb der engen Grenzen des Urheberrechtsgesetzes ist ohne Zustimmung des Verlages unzulässig und strafbar.

Das gilt insbesondere für Vervielfältigungen, Übersetzungen, Mikroverfilmungen und die Einspeicherung und Verarbeitung in elektronischen Systemen.

(C) 1988 Springer-Verlag GmbH Deutschland

Ursprünglich erschienen bei J.B. Metzlersche Verlagsbuchhandlung und Carl Ernst Poeschel Verlag GmbH in Stuttgart 1988 


\section{Der Problemzusammenhang 1}

1. Das Wesen der Grammatik und die Sprachwissenschaft _ 3

2. Die Implikationen der Wesensfrage 6

3. Die Wahrnehmungsmöglichkeiten für das Grammatikproblem

\section{Geschichte des Grammatikbegriffs 14}

1. Die Antike 18

2. Das Mittelalter 21

3. Die frühe Neuzeit 24

4. Das 19. und 20. Jahrhundert 26

\section{Phänomenologie und Grammatik 30}

1. Die Zielsetzungen der Phänomenologie 31

2. Die Grammatik als phänomenologisches Analyseproblem 36

\section{Semiotik und Grammatik 41}

1. Grundlagen und Ziele der Semiotik

a) Pragmatismus und Semiotik 41

b) Vernunftkritik und Zeichenkritik 42

c) Die soziale Dimension der Erkenntnis _ 45

d) Der Peircesche Zeichenbegriff _ 46

2. Semiotische Transformation des Grammatikbegriffs 48

a) Form- und Funktionsgesichtspunkte 48

b) Differenzierungsfunktionen grammatischer Zeichen $\quad 50$

c) Das Inventar grammatischer Zeichen 51

d) Ebenen der Grammatik — 53

3. Betrachtungsmöglichkeiten für sprachliche Zeichen -55

a) Die Abstraktivität sprachlicher Zeichen 56

b) Die Komplexität sprachlicher Zeichen 58

c) Die informative Funktionalität sprachlicher Zeichen 60 
d) Die semantische Selbständigkeit sprachlicher Zeichen 63

4. Lexikalische und grammatische Zeichen 67

\section{Semantik und Grammatik 72}

1. Das Bedeutungsproblem 73

2. Das Problem der grammatischen Bedeutung _ 76

3. Ontosemantische Untersuchungsansätze 78

4. Pragmatische Untersuchungsansätze 83

a) Kognitionssemantik 84

b) Instruktionssemantik 91

\section{Feldgedanke und Grammatik 97}

1. Der Feldgedanke 97

2. Grammatische Felder 100

\section{Evolution und Grammatik 106}

1. Das Evolutionskonzept 107

2. Die kulturelle Evolution 109

3. Die Evolution kultureller Systeme - Recht und Sprache 116

4. Systemzwänge zur Evolution der Grammatik 126

5. Die Evolution der Grammatik in der Ontogenese und Phylogenese _ 130

6. Grammatikgeschichte als Kulturgeschichte 137

a) Die kulturgeschichtliche Dimension grammatischer Formen 138

b) Die kulturgeschichtliche Dimension grammatischer Theorien 145

7. Die Evolution grammatischer Universalien 149

\section{Schrift und Grammatik 154}

1. Die Schriftsprache als eigenständige Realisationsform von Sprache _ 154

2. Das Grammatikproblem in der Schriftsprache 158

3. Die bewußtseinsverändernde Wirkung der Schrift — 166

\section{Logik und Grammatik 173}

1. Klassische Logik und Grammatik 175

2. Semiotische Logik und Grammatik 183

3. Logische und grammatische Ordnungsstrukturen _ 190

4. Die Metaproblematik in der Sprache 193

5. Die Negation als Metaproblem — 200 


\section{Erkenntnis und Grammatik 211}

1. Die Ordnung des Wissens am Leitfaden der Grammatik _ 212

2. Grammatische und kognitive Universalien

3. Das Konzept einer wissenschaftlichen Idealsprache 228

4. Überlegungen zum Verhältnis von Sprache und Denken vor Humboldt _ 235

5. Das Verhältnis von Sprache und Denken bei Humboldt 240

a) Das Sprachkonzept Humboldts 241

b) Das Grammatikkonzept Humbolts _ 244

c) Grammatisch durchgeformte und weniger durchgeformte Sprachen _ 247

6. Die inhaltsbezogene Grammatik 251

7. Die >Allgemeine Semantik « 257

8. Das sprachliche Relativitätsprinzip _ 265

a) Die Grundthese Whorfs — 266

b) Die Relativität grammatischer Formen — 271

c) Die Sprache und die Drei-Welten-Lehre Poppers _ 277

d) Die Funktion der , Zeit in metareflexiven Prozessen — 280

e) Beurteilungen des sprachlichen Relativitätsprinzips _ 282

9. Die Bewertung der sprachlichen Relativität aller Erkenntnis _ 285

a) Das negative Urteil Nietzsches _ 286

b) Das positive Urteil Cassirers 289

10. Grammatische Ordnungsmuster und das Wahrheitsproblem 294

a) Die Korrespondenztheorie — 296

b) Die Widerspiegelungstheorie 300

c) Die Konsensustheorie —_ 306

\section{Hermeneutik und Grammatik 311}

1. Begriff und Erkenntnisinteressen der Hermeneutik _ 312

2. Sprachgefühl und hermeneutischer Zirkel 317

3. Gestaltpsychologie und Gehirnstruktur 325

a) Gestaltpsychologie und grammatische Zeichen 325

b) Gehirnstruktur und Verstehensprozeß 329

4. Informationsschichten in Texten 335

a) Information und Metainformation 336

b) Inhaltsaspekt und Beziehungsaspekt 342

c) Propositionaler Gehalt und illokutionäre Funktion 348

d) Sprachfunktionen und Inhaltskategorien — 354

5. Reliefbildung und Aufmerksamkeitslenkung _ 363

a) Hauptinformation und Nebeninformation 364

b) Perspektivierung von Informationsinhalten 368

c) Abfolge von Informationen — 375 


\section{Vom Sinn grammatischen Wissens 382}

1. Der praktische Sinn grammatischer Kenntnisse -384

2. Der philosophische Sinn grammatischer Reflexionen _ 389

a) Hegels Philosophie der Grammatik _ 391

b) Der Bildungswert grammatischer Studien

3. Der didaktische Sinn des Grammatikunterrichts _ 406

Schlußbemerkungen 415

Anmerkungen 418

Literaturverzeichnis 436

Personenregister 448

Sachregister 451 
Denn wo Gespenster Platz genommen, Ist auch der Philosoph willkommen.

's ist ein Gesetz der Teufel und Gespenster :

Wo sie hereingeschlüpft, da müssen sie hinaus.

Neue Blicke durch die alten Löcher.

Lichtenberg

Es gibt keine Antworten, nur Querverweise.

Bibliotheksweisheit

Fortschritt bedeutet,

heute auf Fragen keine Antwort zu wissen,

die man gestern noch gar nicht gestellt hat.

Bülow

Man glaubt gar nicht, wie schwer es oft ist, eine Tat in einen Gedanken umzusetzen!

Die Tiefe muß man verstecken. Wo?

An der Oberfläche.

von Hofmannsthal

Der Mitgegenwart des Krempeltierchens,

das eine Antwort findet, die zur Frage wird. 
Sich vorzustellen, daß Zeus in einer schwachen oder starken Stunde auch eine Muse der Grammatik gezeugt haben könnte, die alle Erdenkinder zu inspirieren und zu erotisieren vermag, übersteigt wohl selbst die produktive Einbildungskraft der ärgsten Grammatikliebhaber. Realitätsnäher wirkt da wohl schon das allegorische Bild, das Martianus Capella im 5. Jahrhundert entworfen hat, um die Grammatik in der Reihe der sieben freien Künste zu charakterisieren. Er hat nämlich die Grammatik als hochbetagte Greisin dargestellt, die in einem elfenbeinernen Kästchen Messer und Feile mit sich führt, um die Sprachfehler der Kinder chirurgisch zu behandeln. [1]

Ebenso apart wie aufschlußreich ist auch, daß nach Capella die Grammatik von Ägypten über Attika nach Rom gekommen ist und daß sie sich der Abstammung von Osiris rühmt. Osiris ist nämlich in der ägyptischen Mythologie ein geheimnisvoll ambivalenter Gottkönig, der sowohl als Herr der Toten und der Unterwelt gilt als auch als Gott der Fruchtbarkeit, Auferstehung und Erneuerung. In Osiris wird, zusammen mit Isis als der Mutter Erde, die Zeugungskraft der Sonne verehrt. Er wird aber auch als das Land gedeutet, das im Wasser ertrinkt und eben dadurch wieder fruchtbar wird, oder als das Getreidekorn, das in der Erde verschwindet und vergeht und eben dadurch wieder wirksam wird.

Die Dialektik von Tod und Leben, die Osiris mythisch verkörpert, ist für das Verständnis des Phänomens Grammatik sehr aufschlußreich. Wer die Grammatik bewußt kennenlernt, für den stirbt die Sprache als ein unmittelbar handhabbares Mitteilungsmedium; gleichzeitig wird sie aber eben dadurch auch wieder auf neue Weise wahrnehmbar. Einerseits sind deshalb grammatische Reflexionen untergründig immer mit einer melancholischen Trauer um den Verlust des naiv-spontanen Sprachgebrauchs verbunden, der zugleich auch einen Verlust des Urvertrauens zur Sprache beinhaltet. Andererseits entsteht aber eben dadurch auch wieder eine erwartungsvolle Spannung auf neue Erfahrungen an und mit der Sprache.

Die Möglichkeit und der Zwang, seinen Sprachgebrauch grammatisch zu kontrollieren, kann den eigenen Sprachformen etwas von ihrer vitalen Kraft nehmen, aber ihnen zugleich auch die Festigkeit mechanischer Ordnungen verleihen, wenn nicht gar ein Stück Totenstarre. Insbesondere im schriftlichen Sprachgebrauch ermöglicht die bewußte grammatische Kontrolle von Äußerungen ein Maß an semantischer Präzision, das die Sprache erst zu einem wirklich autonomen Sinnbildungsinstrument werden läßt.

Wer sich von der Grammatik faszinieren läßt, der scheint auf den ersten Blick dem prallen Leben ganz entrückt zu sein. Zumindest scheint er nicht mehr durch das beflügelt zu werden, was üblicherweise beflügelt. Wer sich auf die Grammatik einläßt, 
der hat sich gewollt oder ungewollt immer schon irgendwie auf die Philosophie eingelassen. Ebenso wie die Philosophie gehört offenbar auch die Grammatik zu den Nachtvögeln aus dem Horste der Minerva. Noch später als die philosophische Eule, die nach Hegels Überzeugung erst in der Dämmerung ihren Flug beginnt, scheint die grammatische Eule ihren Flug begonnen zu haben bzw. beginnen zu können. Deshalb ist es wohl auch nicht verwunderlich, wenn die Grammatik vielen eher als Schreckgespenst der Nacht erscheint denn als heiliger Vogel der Weisheitsgöttin.

Die Art und Weise, in der wir üblicherweise die Grammatik kennenlernen, macht es sicherlich schwer, ihr gegenüber in Liebe zu entbrennen. Vermutlich würden wir eher dem Teufel als ihr eine Kerze anzünden. Dennoch sollten wir uns aber bemühen, die Grammatik nicht mit dem ausgestopften Vogel zu identifizieren, den die Grammatiker zu Demonstrationszwecken aus ihr gemacht haben. Wir sollten sie auch nicht mit den grauen Bildern verwechseln, die in dicken Folianten von ihr gemalt worden sind. Vielmehr sollten wir uns anstrengen, sie dort kennenzulernen, wo sie lebt und fliegt. Vielleicht lassen sich dann im vermeintlich nachtgrauen Gefieder der grammatischen Eule doch noch einige bunte Federn entdecken bzw. an ihren Lebens- und Flugformen Eigenschaften, die uns in Erstaunen zu versetzen vermögen. Gerade weil die grammatische Eule sich dem Blickfeld unserer Alltagsgeschäfte entzieht und gerade dann aktiv wird, wenn wir keinerlei Aktivität mehr vermuten, kann sie zu einem Faszinosum für handverlesene Liebhaber werden.

Über die Grammatik als Inventar sprachlicher Ordnungsformen ins Staunen zu geraten, das nach Sokrates[2] ja den Anfang aller Philosophie ausmacht, ist im Prinzip eigentlich nicht allzu verwunderlich. Ein etwas genauerer Blick zeigt nämlich schnell, daß die grammatischen Ordnungsformen der Sprache zugleich auch geistige Ordnungsformen sind, durch die unser Denken in der Wolle eingefärbt wird, ohne daß wir uns dessen im alltäglichen Leben recht bewußt werden. Die Grammatik kann insbesondere deswegen zu einem genuinen Gegenstand philosophischen Staunens werden, weil dieses sich im Gegensatz zu dem üblichen Staunen dadurch auszeichnet, daß es nicht durch etwas Exorbitantes ausgelöst wird, das unsere natürliche Fassungskraft übersteigt, sondern gerade durch das, was im alltäglichen Bewußtsein völlig natürlich, selbstverständlich, ja trivial erscheint.

Über das scheinbar Selbstverständliche ins Staunen zu geraten ist nun allerdings nicht jedermanns Sache; und über die Grammatik ins Staunen zu geraten ist sicher wenigen vorbehalten. Staunen wird der unbefangene Zeitgenosse wohl weniger über die Grammatik selbst als darüber, daß jemand über die Grammatik ins Staunen geraten kann. Über das eigentlich Selbstverständliche in Verwunderung zu geraten wird aus der Perspektive des praktischen Lebens meist als Zeichen von Weltfremdheit und Skurrilität gewertet, obwohl es weder leicht noch unproblematisch ist.

Leicht ist das philosophische Staunen deswegen nicht, weil es nur dann zu verwirklichen ist, wenn wir unser Alltagsbewußtsein aufgeben und uns einen neuen Ausgangspunkt für unsere Wahrnehmungen suchen. Nur dann haben wir nämlich die Chance, dasjenige in den Blick zu bekommen, was wir praktisch verwenden, ohne es theoretisch zu durchschauen, bzw. das, was wir zu beherrschen glauben, obwohl wir auch von ihm beherrscht werden. Unproblematisch ist das philosophische Staunen vor 
allem deshalb nicht, weil dabei die stabilisierenden Grundaxiome unseres Denkens leicht ins Wanken geraten können. Worüber man staunen kann, das büßt seinen natürlichen Geltungsanspruch ein, eben weil man es sich auch ganz anders denken kann, als es tatsächlich ist. Was man sich aber auch anders denken kann, das bedarf der Rechtfertigung, wenn es weiterhin eine verpflichtende Norm bleiben soll.

Über grammatische Formen kann man philosophisch sowohl staunen, daß es sie überhaupt gibt, als auch darüber, daß es sie in den einzelnen Sprachen so gibt, wie es sie gibt. Gerade weil grammatische Formen eigentlich gar nichts Spektakuläres an sich haben, dürfen sie unsere philosophische Aufmerksamkeit beanspruchen. Sie bieten nämlich dem Denken die Chance, sich vom Alltagsbewußtsein mit seinem Interesse für die Welt der Sachen zu lösen und seine Aufmerksamkeit ganz auf die Mittel zu konzentrieren, mit deren Hilfe wir uns in ihm die Welt der Sachen konkretisieren und repräsentieren.

Die hier vorgetragenen Überlegungen sollen dazu anregen, etwas sehen zu lernen, was üblicherweise nicht sichtbar ist, und über etwas ins Staunen zu geraten, was es für das sachbezogene Alltagsdenken gar nicht gibt. Sich an Gegenständen abzumühen, die im praktischen Lebensvollzug gar nicht zu existieren scheinen und die wir uns erst mühsam vergegenständlichen müssen, wird sicher vielen als ein Symptom kauziger Absonderlichkeit erscheinen oder gar als ein magisch-circensischer Trick, bei dem man an einem Seil hinaufklettert, das man sich vorab selbst in die Luft geworfen hat. Den meisten wird das Studium der Grammatik wohl immer eine dürre Weide bleiben. Dennoch kann ihm aber auch der Reiz eines geistigen Spiels zuwachsen, das eine besondere anthropologische Funktion und Dignität hat.

Es scheint nämlich ein menschliches Grundbedürfnis zu sein, etwas über die Genese, Geschichte, Kraft und Funktion der Mittel in Erfahrung zu bringen, die der menschliche Geist im Laufe seiner Entwicklung hervorgebracht hat, um sich selbst zu entfalten und zu konkretisieren. Grammatische Reflexionen sind nicht nur eine Luxusbeschäftigung menschlicher Geistestätigkeit an lauen Sommerabenden, sondern auch ein Stück geistiger Grundhygiene, die man nicht ungestraft vernachlässigen sollte. Grammatische Reflexionen werden immer einen purgatorischen Charakter behalten, weil sie ohne Arbeit, Mühe und Schweiß nicht denkbar sind. Die Katarakte der ersten Begeisterung versiegen deshalb bei ihnen auch immer wieder schnell im Sande. Gleichwohl kann sich in ihnen aber auch die spielerische Lust geistiger Akrobatik entzünden. Dann stehen die Chancen nicht schlecht, daß aus ihnen nicht nur eine Amateur, sondern sogar ein amator der Grammatik hervorgeht.

Die Philosophie der Grammatik darf Ziele verfolgen, die quer zu denen liegen, die üblicherweise in Grammatiken bzw. grammatischen Theorien angestrebt werden. In ihr muß die Grammatik weder praktisch noch theoretisch serledigt darf sie den Wunsch anregen, die Grammatik immer intimer kennenzulernen und sich immer mehr in sie zu verwickeln; denn die schönsten Gefangenen sind ohnehin diejenigen, die einen selbst gefangen nehmen. Und falls es jemandem wie dem Lehrling zu Sais gehen sollte, wenn er den Schleier der Grammatik zu lüften wagt - es wäre kein Fehler.[3] 